\title{
10
}

\section{Medical Care of the Surgical Patient}

\section{Mel P. Daly}

Family physicians are frequently called on for consultations and medical management of patients who require surgical procedures. Advances in surgical techniques and anesthesia have significantly reduced the risk of death and serious morbidity from intraoperative and postoperative complications. ${ }^{1}$ Factors that increase the risk for adverse outcomes have been more clearly defined, and interventions to address and treat known risk factors before surgery have contributed to declines in morbidity and mortality. Notwithstanding these advances, up to $20 \%$ of all surgical patients have at least one perioperative complication. Most surgical morbidity and mortality occurs as a result of cardiac (5\%), pulmonary (10\%), or infectious complications $(15 \%){ }^{2}$

There are 25 million patients who undergo noncardiac surgery in the United States annually. Up to 7 million of them have cardiac disease or are at risk of developing cardiac disease during the operative period. About 50,000 patients sustain perioperative myocardial infarctions (MIs); $50 \%$ die as a result of their MI. Whereas cardiac problems are the major cause of mortality, most morbidity results from pulmonary complications, sepsis, or renal failure. Overall risk is related to individual patient factors (coexistent medical illness, age, pathology requiring surgery) and the type and urgency of the surgical procedure. ${ }^{3}$

The goal of the medical consultant is to identify prohibitive or potentially treatable risk factors so that the patient can be taken to the operating room in the best possible condition within the time avail- 
able. Prior to surgery, patients, families, and surgeons should be aware of the potential medical risks of the procedure and if this risk can be reduced by preoperative interventions. It is imperative that family physicians become involved in the medical care of their patients during this most physiologically stressful time of their life.

\section{Preoperative Assessment}

\section{History}

The preoperative medical history should be comprehensive and focus on uncovering factors that may affect outcome. Previous surgery or anesthetic mishaps should be documented. It is important to take a menstrual history to prevent elective surgery on a pregnant woman inadvertently. For pediatric patients, particular attention must be paid to recent or current infectious conditions to ensure that they are infection free at the time of surgery. Seriously chronically ill pediatric patients with congenital anatomic anomalies (especially those children with cardiac disease) should be referred to specialists, when appropriate, prior to surgery.

A list of prescription and nonprescription medications should be obtained. Cardiac medications (digoxin, antiarrhythmic agents), antihypertensives (beta-blockers and alpha-blockers), major tranquilizers, and monoamine oxidase inhibitors can result in life-threatening arrhythmias. All currently taken medications should be documented and strategies developed for their use before, during, and after surgery. Patients should be asked about their use of corticosteroids within the previous 6 months to a year, as there are reports of patients failing to mount an adequate intraoperative stress response to surgery as a result of iatrogenic hypothalamic-pituitary-adrenal axis suppression. ${ }^{4}$ All patients should be asked about their use of alcohol, cigarettes, and recreational drugs.

A major determinant of outcome for patients undergoing noncardiac operations is baseline functional capability. This is especially true for older patients, and patients with cardiac and pulmonary conditions. All patients should be asked about their daily routines and how well they are able to perform activities of daily living. Patients who can climb a flight of stairs without difficulty or who can mow their lawn, play golf without a cart, or do housework are likely to have good physiologic reserve and will usually tolerate most surgical procedures. For older patients, this assessment is also likely to be useful in predicting how much help will be required after surgery that 
will result in functional impairment after discharge (e.g., hip or knee replacement surgery, or trauma surgery).

\section{Physical Examination}

A comprehensive physical examination is always indicated. Anesthesiologists appreciate knowing about a deviated nasal septum, loose teeth, and the patient's ability to open his or her mouth. The examiner should pay particular attention to the cardiac and pulmonary systems. The heart rhythm, presence of significant murmurs, and added sounds (particularly an $\mathrm{S}_{3}$ ) should be noted. It is important to note signs of congestive heart failure (rales, jugular venous pressure, edema) and aortic stenosis (murmur, pulse pressure). A careful respiratory examination, observing for lung expansion and diaphragmatic excursion and listening for wheezing or rhonchi, may identify the presence and severity of emphysema, asthma, or chronic obstructive lung disease. Patients with rheumatoid arthritis have a high incidence of atlantoaxial joint involvement and may be at risk of spinal cord compression with hyperextension during intubation. Range of motion should be tested and cervical spine radiographs considered. A mental status examination should be done for all elderly patients prior to surgery, because of the high incidence of postanesthesia delirium among elderly surgical patients.

\section{Laboratory Testing}

Many studies cite the lack of data supporting the routine use of laboratory testing before surgery. Many screening tests are ordered, but few are abnormal and rarely is surgery or anesthesia changed as a result. ${ }^{5}$ This is especially true for patients who are to have low-risk surgical procedures (e.g., cataract surgery, breast biopsy, podiatric procedures, outpatient procedures, hernia repair). 6 Thus, routine preoperative laboratory testing is not justified; selective testing should be done instead if there are specific clinical indications. From a primary care perspective, routine testing may be indicated if a broader focus on long-term, otherwise neglected health care needs is appropriate. In many instances surgery is necessary and may be the only reason for someone to see a physician. This may present an opportunity for primary care assessment and maintenance.

It has been estimated that it costs in excess of $\$ 2$ million for screening tests to identify an abnormal prothrombin time if it is not suspected by the history and physical examination. It is unusual to detect renal abnormalities on preoperative laboratory testing unless there is a suspicion based on risk factors for renal insufficiency (e.g., 
diabetes mellitus, hypertension, use of nephrotoxic medications). Similar data have revealed low yields for detecting abnormal results by doing a routine complete blood count (CBC), urinalysis, and liver function tests in asymptomatic patients. Thus laboratory testing must relate to the medical history, the proposed surgical procedure, and the potential for morbidity and mortality. ${ }^{7}$

In most centers patients are required to have a $\mathrm{CBC}$ prior to surgery, yet a strong argument can be made not to obtain this test, especially for men under age 60 , as the incidence of anemia is low. Surgeons are unwilling to operate without knowing their patient's hemoglobin level, prothrombin time (international normalized ratio, INR), and partial thromboplastin time (PTT) because of potential bleeding risks; however, there is little indication for doing these tests unless there is a specific indication.

There is a high incidence of unsuspected abnormal electrocardiograms (ECGs) among presurgical patients, yet whether it changes the anesthesiologist's approach to surgery has not been studied. Because most deaths after surgery result from cardiac complications, it is worthwhile to order an ECG prior to surgery for people over age 40. After age 70, a measure of renal function (blood urea nitrogen, creatinine) is indicated because of age- and disease-related changes in creatinine clearance.

The likelihood of finding abnormal laboratory tests is increased in patients with underlying pathologic conditions and those taking prescription medications. Patients who have a history of bleeding disorders or bruiseability/excessive bleeding or who are currently taking anticoagulant medications should have a prothrombin time, INR, PTT, and platelet count determined. Those with renal disease should have their renal function measured, and patients with diabetes or who are currently taking corticosteroids should have serum electrolyte and glucose levels determined. Patients taking digitalis should have serum digoxin and potassium levels measured. Previously ordered laboratory tests rarely need to be repeated; tests done up to 4 months prior to surgery that were normal are rarely abnormal on repeat testing and usually do not affect anesthesia or surgical outcomes. Thus it is reasonable to accept laboratory testing and ECGs done within 4 months of elective surgery. ${ }^{8}$

\section{Preoperative Report}

Surgery should be postponed if a patient's medical condition can be improved and surgery safely delayed (elective and semielective procedures). If, in the opinion of the medical consultant, surgery ought 
to be postponed, it should first be communicated to the surgeon. The written preoperative report should identify medical conditions that place the patient at increased risk of adverse outcomes and make suggestions about how risk may be reduced in the time available.

It is tempting to make recommendations about the type and route of anesthesia. There is, however, little evidence to suggest that the type or route of anesthesia is important for predicting adverse outcomes. ${ }^{9}$ General anesthetic agents are myocardial suppressants and peripheral vasodilators. Spinal anesthesia induces a sympathectomy at the level at which it is administered and causes levels of hypotension similar to those seen with general anesthetic agents. Furthermore, spinal anesthesia induces motor muscle paralysis that may interfere with forced expiration. Patients may have a heightened level of anxiety because they are awake, which increases myocardial oxygen demand. There is a greater likelihood of aspiration pneumonitis among patients given spinal anesthesia because the airway is unprotected. Occasionally, particularly in elderly patients, it is not possible to administer spinal anesthesia because of thoracolumbar spondylosis. Thus if a patient is "cleared" for spinal anesthesia and the anesthesiologist is technically unable to administer spinal anesthesia, general anesthesia may be the only option.

\section{Assessment of Risk}

The risk of mortality or morbidity is low and is individualized and related to the presence and severity of comorbid medical illnesses (especially cardiac and pulmonary pathology), the surgical procedure, and whether the procedure is emergent or elective. The risk of an adverse outcome can be estimated in a number of ways. The American Society of Anesthesiologists (ASA) physical status classification, first described during the $1950 \mathrm{~s}$, is widely used today. ${ }^{10}$ Patients are classified based on their physical health status to somewhat qualitatively determined classes, ranging from class 1 (healthy) to class 5 (moribund). In large studies describing outcomes of thousands of operations, the ASA classification system has proved useful for predicting risk of complications and death. Limitations of the ASA system are that assignment of risk is subject to observer bias, and the inherent risk of the surgical procedure is not considered. Emergency operations are associated with a two- to fourfold risk of adverse outcomes when compared to elective surgery. True surgical emergencies (perforated organ, peritonitis, trauma, aortic aneurysm) rarely allow time for a comprehensive preoperative medical evaluation. In most other instances, the risk of adverse outcomes can be estimated 
by assessing clinical predictors in the context of the hemodynamic physiologic stress (alterations in heart rate, blood pressure, volume, hemoglobin, oxygenation, thrombogenicity) of the proposed operation. Major surgery can be long (intrathoracic, vascular, or neurosurgical procedures) and associated with a greater likelihood of adverse outcomes. Moderate-risk operations include extremity operations lasting 2 hours or longer (e.g., orthopedic surgery), and surgery that is less likely to result in adverse medical outcomes includes distal extremity operations, hernia repair, thoracoscopy, and transurethral resection of the prostate (Table 10.1).

Another commonly used risk assessment instrument is the Goldman Cardiac Risk Index, which was first published during the late

\section{Table 10.1. Surgical Procedure Risk}

High risk (reported cardiac risk $\geq 5 \%$ )

Coronary artery bypass graft surgery

Pneumonectomy

Trauma surgery

Neurosurgery

Major vascular procedure

Ruptured abdominal viscus

Emergency surgery

Anticipated prolonged surgery, with hemodynamic instability

Moderate risk (reported cardiac risk usually $\leq 5 \%$ )

Abdominal surgery (open cholecystectomy, colon resection, etc.)

Orthopedic surgery

Urogynecologic surgery (prostatectomy, hysterectomy, cesarean section)

Splenectomy

Cancer staging procedures

Peripheral vascular procedures (endarterectomy, femoral-popliteal bypass)

Prostate surgery

Low risk (generally $<1 \%$ )

Cataract surgery

Podiatry procedures

Endoscopy and biopsy

Breast biopsy

Mastectomy

Herniorrhaphy

Vasectomy

Appendectomy

Dermatologic procedures

Source: Modified from Eagle et al, ${ }^{16}$ with permission. 
1970s and has been widely used subsequently. ${ }^{11}$ More than 1000 patients admitted to Massachusetts General Hospital for surgical procedures underwent comprehensive preoperative physical assessments and were followed postoperatively to identify major cardiac complications and death. A multivariate discriminate risk analysis identified factors significantly associated with these outcomes, and each risk factor was assigned a weighted point score based on its relative association with adverse outcomes. Based on point scores, patients were retrospectively assigned to a level of risk associated with serious cardiac complications (ventricular tachycardia, death, MI, pulmonary edema) (class I, $0.7 \%$, to class IV, $22.0 \%$ ) and mortality (class I, $0.2 \%$, to class IV, $50.0 \%$ ). Factors not found to increase the risk of serious cardiovascular morbidity and mortality were controlled hypertension, the presence of an $\mathrm{S}_{4}$, diabetes mellitus, hyperlipidemia, and stable angina pectoris. The latter was defined as angina that had not changed over the previous year in patients able to ambulate a distance of two blocks. Some of these factors (congestive heart failure and general medical condition) are potentially reversible preoperatively.

Lower reinfarction rates for patients operated on within 6 months of the original MI have been reported more recently and reflect an increased awareness of risk and more experience using invasive hemodynamic monitoring, pressors, and beta-blockers. ${ }^{12}$ Perhaps more important for estimating the risk of reinfarction is the extent of prior myocardial damage and whether the patient has myocardium that is at risk because of coronary artery stenosis. Patients who have had non-Q-wave MIs may be at a greater risk of reinfarction than patients who have survived a transmural infarction. These patients frequently have borderline zones of potential ischemia that may be in jeopardy during anesthesia. Symptom-linked exercise testing to assess the extent of previous damage and identify at-risk myocardium is indicated in all patients who have had recent MIs requiring noncardiac surgery.

More recently published cardiac risk indices have taken these and other factors into consideration. Subsequent modifications (Table 10.2) of the Goldman Risk Index and includes point ratings for unstable angina (class III angina after one to two blocks of ambulation; class IV angina at rest) and critical aortic stenosis. ${ }^{13}$ Each of the published cardiac risk indices has been studied and shown to accurately predict the risk (class III or IV) of having an adverse cardiac outcome (pooled data: $16 \%$ for class III and $56 \%$ for class IV). A higher than expected serious complication rate for class I and II patients has been reported among those operated on for abdominal aortic 
Table 10.2. Cardiac Risk Assessment (Modified Multifactorial

Risk Index)

Criteria, points

Coronary artery disease, 10

Myocardial infarction $\leq 6$ months

Myocardial infarction $>6$ months

Canadian Cardiovascular Society angina

Class 3, 10

Class 4, 20

Unstable angina within 3 months, 10

Alveolar pulmonary edema

Within 1 week, 10

Ever, 5

\section{Valvular disease}

Critical aortic stenosis, 20

\section{Arrhythmias}

Sinus plus atrial premature beats or rhythm other than sinus on preoperative ECG, 5

More than $5 \mathrm{PVCs} / \mathrm{min}$ at any time prior to surgery, 5

\section{Medical status, 5}

Poor general medical status

$\mathrm{PO}_{2}<60$ or $\mathrm{PCO}_{2}>50$

$\mathrm{K}<3.0$ or $\mathrm{HCO}_{3}<20 \mathrm{mEq} / \mathrm{L}$

BUN $>50 \mathrm{mg} / \mathrm{dL}$ or creatinine $>3 \mathrm{mg} / \mathrm{dL}$

Abnormal SGOT

Chronic liver disease

Bedridden due to noncardiac cause

Age $>70$ years, 5

Operation: emergency, 10

Source: Modified from American College of Physicians, ${ }^{17}$ with permission.

ECG = electrocardiogram; PVCs = premature ventricular contractions; $\mathrm{BUN}=$ blood urea nitrogen; SGOT = serum glutamate oxolic acid.

aneurysms, probably reflecting a high incidence of asymptomatic cardiac disease among these patients, resulting in their being misclassified as low-risk class I patients. ${ }^{14}$

\section{Cardiac Risk}

\section{Coronary Artery Disease}

Patients with a history of a documented MI have a greatly increased likelihood of having an intra- or postoperative cardiac complications 
(see Chapter 2). This is especially true for patients who have sustained their infarction within 3 months of their noncardiac surgery. Patients who have sustained a recent MI and who have evidence of residual ischemic risk (unstable or severe angina, poorly controlled ischemic-mediated congestive heart failure, or severe valvular heart disease) should be considered at prohibitively high risk for adverse perioperative cardiac complications. These patients should be referred to cardiologists for further evaluation and potential cardiac surgical interventions prior to considering noncardiac elective surgery. ${ }^{15}$

Patients with mild angina, prior MI (older than 1 month), history of congestive heart failure, diabetes mellitus, and elderly patients should be classified at intermediate risk for having perioperative cardiac-related complications. Patients with underlying peripheral vascular disease should also be considered at intermediate risk because of the high incidence of coexisting cardiac pathology (often "silent"). ${ }^{16}$ Patients without cardiac disease have a low incidence of postoperative MI and other cardiac complications (Table 10.3).

An important component of the preoperative cardiac evaluation for all patients is an assessment of how well a patient can perform functional activities. This may be helpful in deciding whether further cardiac testing is necessary, especially for intermediate-risk patients who are scheduled to have major surgery. Patients who can participate in strenuous activities such as running, playing basketball, or longdistance swimming have excellent functional reserve capacity, while sedentary patients who have difficulty carrying groceries up a flight of stairs have poor functional capacity. Patients with excellent functional capacity usually can tolerate major surgery, while patients with poor functional capacity are at much greater risk for adverse outcomes. Patients with moderate functional capacity should be further evaluated if they have intermediate- or high-risk clinical profiles or if they are to have major surgery.

Further testing and recommendations about how best to proceed can be made by carefully evaluating the preoperative clinical risk profile, functional capacity, and the proposed procedure. This is outlined by the American College of Cardiology/American Heart Association (ACC/AHA) Task Force, which suggests a progressive stepwise approach to preoperative cardiac assessment (Fig. 10.1).

The goal of the preoperative assessment is to evaluate not only the risk of the immediate surgery but also the long-term cardiac risk. Thus this may be an ideal time to consider further testing (noninvasive or invasive) if this is likely to improve outcome. Noninvasive testing (see below) should be considered for patients who are at intermediate clinical risk and are to undergo a major procedure. Exer- 


\section{Table 10.3. Clinical Predictors of Increased Perioperative}

\section{Cardiac Risk}

\section{High risk}

Recent myocardial infarction (within 1 month)

Congestive heart failure (unstable)

Valvular heart disease (esp. aortic stenosis)

Unstable angina

Significant cardiac arrhythmias (ventricular tachycardia with ischemia, supraventricular arrhythmias with uncontrolled ventricular response)

\section{Intermediate risk}

History of myocardial infarction

Stable congestive heart failure

Stable/mild angina pectoris

Diabetes mellitus

Age over 70 years

Rhythm other than normal sinus rhythm on the preoperative ECG

Uncontrolled hypertension

Peripheral vascular disease

Mitral valve prolapse \pm regurgitation

\section{Low risk}
Age over 70 years
Controlled hypertension
History of cerebrovascular disease
Cardiac murmur
"Minor" ECG abnormalities (premature atrial contractions, nonspecific STT changes)
Controlled atrial fibrillation
History of stroke
Low functional capacity

STT $=$ serial thrombin time.

Source: Modified from Eagle et al, ${ }^{16}$ with permission.

cise stress testing, echocardiography (resting, stress), or perfusion imaging (dipyridamole, dobutamine) can further assist in stratifying risk; however, there is as yet no evidence that this type of testing improves perioperative care. Invasive testing (arteriography) should be reserved for high-risk patients with suspected left main disease, triple vessel disease, or unstable angina in whom angioplasty or coronary artery bypass grafting would be indicated. Patients at low risk should have no further testing. ${ }^{17}$

The peak time of occurrence of postoperative MI is 3 to 6 days after surgery; it is due to increased activity, pain, and shifts of third- 


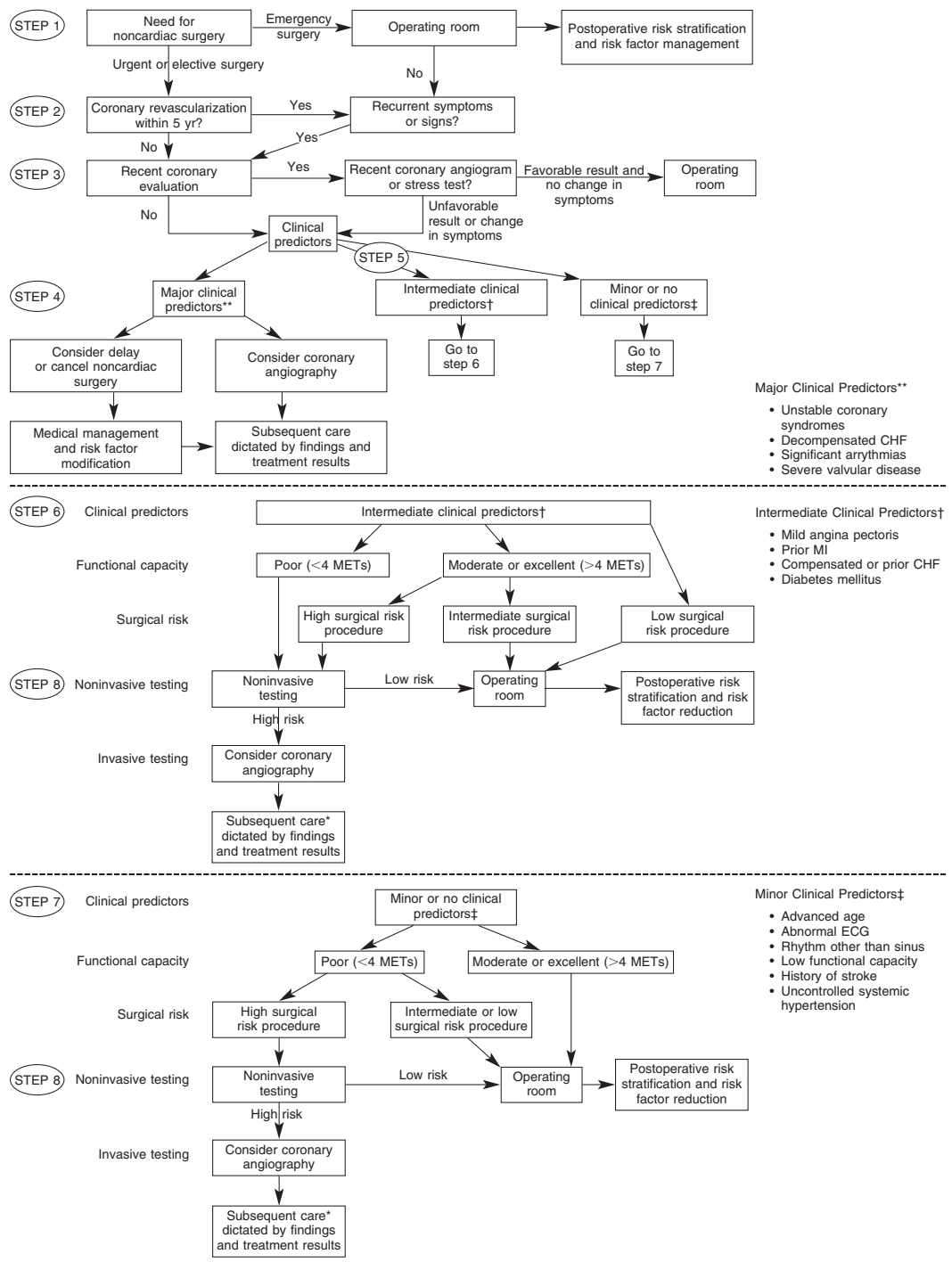

Fig. 10.1. Stepwise approach to preoperative cardiac assessment. Steps are discussed in text. *Subsequent care may include cancellation or delay of surgery, coronary revascularization followed by cardiac surgery, or intensified care. (From Eagle et al, ${ }^{16}$ with permission.) 
space fluid. Most postoperative MIs are "silent," perhaps accounting for the high mortality (up to 50\%) among patients who sustain postoperative MIs. Patients who have had previous coronary artery bypass graft $(\mathrm{CABG})$ surgery have a low incidence of infarction when subjected to further noncardiac procedures. ${ }^{18}$ The restenosis rate after CABG surgery (native or saphenous graft) increases with time after the original surgery. This is also true for patients who have had percutaneous transluminal coronary angioplasty (PTCA). Asymptomatic patients who have undergone coronary artery bypass grafting within the last 5 years and patients who have had coronary evaluation (cardiac stress testing) within the last 2 years and are clinically stable require no further cardiac testing prior to noncardiac surgery. ${ }^{16}$

Surgical stress can lead to increased circulating levels of catecholamines that can result in arrhythmias and atherosclerotic plaque rupture. One study suggests that the use of beta-blockers can reduce the incidence of perioperative myocardial ischemia, and over time (6 months to 2 years) the incidence of MI, congestive heart failure, and death. ${ }^{19}$ While these data are less than conclusive, it seems prudent that all patients (unless there is a contraindication) with an intermediate or high risk for cardiac complications should be given therapeutic doses of beta-blockers prior to surgery. ${ }^{20}$

In certain situations, preoperative intensive care, intraoperative infusion of nitroglycerine, transesophageal echocardiographic monitoring, perioperative use of pulmonary artery catheterization, and surveillance for perioperative MI may be indicated.

\section{Congestive Heart Failure}

The presence of preoperative congestive heart failure (CHF) has repeatedly been shown to be associated with a risk of postoperative CHF (see Chapter 5). Ideally, patients who present with CHF should be hemodynamically stable for a period of about 2 weeks before surgery. ${ }^{21}$ Invasive (Swan-Ganz catheter) monitoring and treatment may be indicated for patients who have CHF and require emergent or urgent major surgical procedures. Patients with low left ventricular ejection fractions (EFs) are more likely to develop postoperative CHF, whereas those with EFs of $50 \%$ or more have low complication rates, even if they have had a history of CHF. The presence of jugular venous distention, current pulmonary edema, or an $\mathrm{S}_{3}$ gallop places a patient at risk for perioperative cardiac complications. If possible, surgery is postponed until the CHF has been treated with a goal of euvolemia for a period of 2 weeks or more prior to surgery. 


\section{Hypertension}

Poorly controlled hypertension is associated with perioperative cardiac complications, blood pressure lability, and renal failure (see Chapter 1). Severe hypertension may occur especially during induction of anesthesia, intubation, and emergence from anesthesia. Diastolic blood pressure readings of more than $120 \mathrm{~mm} \mathrm{Hg}$ and systolic blood pressures of more than $200 \mathrm{~mm} \mathrm{Hg}$ are associated with a greater likelihood of adverse cardiac outcomes. ${ }^{22}$ Patients with wellcontrolled hypertension are not at increased risk during surgery. Patients taking antihypertensive medications such as beta-blockers, angiotensin-converting enzyme (ACE) inhibitors, clonidine, and calcium channel blockers show attenuated blood pressure responses to intubation and induction of anesthesia. Of greater importance for patients with mild to moderate hypertension is the effect of anesthesia on mean arterial blood pressure (MAP) levels. Large reductions in MAP ( $>30 \%$ for more than 10 minutes) are associated with a greater likelihood of intraoperative MI, CHF, and renal insufficiency. The goal of treatment prior to surgery is to achieve consistent systolic blood pressure readings below $170 \mathrm{~mm} \mathrm{Hg}$ and diastolic blood pressures below $110 \mathrm{~mm} \mathrm{Hg}$.

\section{Cardiac Arrhythmias}

The significance of cardiac arrhythmias identified preoperatively is somewhat controversial (see Chapter 3), as it has become clear that the presence of nonsustained premature ventricular contractions (PVCs) is not a risk factor for ventricular tachycardia or sudden death unless associated with underlying cardiac ischemia (suggesting the presence of severe coronary artery disease). ${ }^{23}$ Prophylaxis with antiarrhythmic medications is indicated for patients with sustained ventricular tachycardia, especially if they have had a recent MI. Atrial fibrillation is an important rhythm during the perioperative period, because when the ventricular response rate is rapid, the ability to increase cardiac output is compromised. This situation occurs because of reduced left ventricular filling volume as a result of a shorter diastolic filling time and loss of the presystolic "atrial kick." Newonset atrial fibrillation, particularly in patients with fixed outflow tract obstruction (aortic stenosis, asymmetric septal hypertrophy) may result in severe CHF and an inability to increase cardiac output. Thus control of the ventricular rate becomes important during anesthesia to allow adequate end-diastolic filling volumes and cardiac output. There is a higher incidence of atrial fibrillation during anesthesia in 
patients who undergo long surgical procedures, those with thoracic or neurosurgical procedures, and the elderly. Digoxin, beta-blockers, or calcium channel blockers should be continued up to and including the day of surgery. Most patients undergoing anesthesia (especially those with a history of arrhythmias) develop some kind of arrhythmia on induction of anesthesia, usually isolated occasional ectopic ventricular or atrial beats. Supraventricular arrhythmias may exacerbate underlying cardiac disease by increasing myocardial oxygen demand. These arrhythmias may also be a manifestation of noncardiac problems such as electrolyte abnormalities, or infection.

It is rare that patients need to have a pacemaker inserted prior to a major surgical procedure. ${ }^{24}$ Placement of a pacemaker should be considered in patients who have long sinus pauses, high-grade second-degree heart block, or complete heart block. There is no indication for pacemaker placement in patients with first-degree atrioventricular (AV) block or asymptomatic bifascicular block, as these patients rarely develop complete heart block. The use of the electrocautery machine during surgery may interfere with "demand" pacemaker function; if the earth lead is placed away from the pacemaker magnet and the surgeon administers short bursts of electrocautery, this effect is reduced. However, it may be necessary to convert the pacemaker function to the fixed-rate mode when "demand" pacemaker responses are suboptimal.

\section{Valvular Heart Disease}

Valvular heart disease (especially hemodynamically significant aortic stenosis) is associated with an increased risk for perioperative mortality $^{25}$ (see Chapter 4). Severe and symptomatic aortic stenosis may require valve replacement or valvuloplasty if high-risk noncardiac surgery is to be done safely. Mitral stenosis is not associated with increased mortality, but these patients are sensitive to preload volume changes especially when tachycardia results in a reduction in diastolic filling time. Patients with aortic regurgitation and mitral regurgitation are less sensitive to volume changes but require adequate left ventricular contractility, as regurgitation takes place during both diastole and systole. Bradycardia is less well tolerated because of increased potential for regurgitation during prolonged diastole. Prosthetic valves increase the risk of bacterial endocarditis and thrombotic complications. Asymmetric septal hypertrophy may be difficult to manage perioperatively because the degree of outflow tract obstruction is variable. Certain medications exacerbate outflow tract obstruction; diuretics and nitrates reduce preload and intravascular volume; inotropes may increase obstruction because of increased 
contractility; and tachycardia may cause similar effects because of decreased diastolic filling time.

\section{Peripheral Vascular Disease}

There is a high incidence of occult coronary artery disease among patients with peripheral vascular disease (PVD) (see Chapter 2). Up to $60 \%$ have severe coronary artery disease; many have no clinical symptoms and frequently have normal ECGs. Patients with PVD have a high incidence of postoperative mortality, mostly because of intraoperative and postoperative MIs. ${ }^{26}$ Additional cardiac testing should be considered for patients with PVD scheduled for major surgery. ${ }^{27}$ Exercise stress testing (EST), if positive, can identify patients at increased risk of having a cardiac event, but the positive predictive value is low. The negative predictive value is high (i.e., a negative test makes it unlikely that a patient will develop a postoperative cardiac complication). Many patients cannot tolerate EST because of intermittent claudication, deconditioning, rest ischemia, or amputations. Furthermore, many patients with PVD have ECG changes of the left bundle branch block or left ventricular hypertrophy, or they are currently taking digoxin, making exercise test ECG readings uninterpretable.

Evidence has suggested that perioperative ECG monitoring may be useful in patients with PVD. ${ }^{28}$ Preoperative ischemia and silent ischemia are good predictors of perioperative and postoperative ischemia and cardiac events. Furthermore, patients without signs of ischemia preoperatively are unlikely to have ischemic events. Again, the positive predictive value of silent ischemia is low because few patients with PVD and positive tests develop postoperative cardiac complications.

Perhaps even more useful is chemical stress testing using nuclear medicine imaging or echocardiography. ${ }^{29}$ Dipyridamole is a coronary vasodilator; hence, stenotic arteries do not dilate, and infarcted areas remain unperfused. In normal hearts thallium is evenly distributed throughout the myocardium, with no distribution distal to stenosed arteries. These areas show up as "cold spots" on thallium imaging studies. If thallium redistributes on delayed imaging (4 hours later) to a previously imaged "cold spot," it may be an indication of underperfused myocardium. Thus redistribution on late imaging with thallium may be an important factor for determining the risk of intraoperative ischemia in a patient with PVD. The advantages of dipyridamole thallium testing are that patients do not have to exercise and increase their target heart rate, and there are few complications associated with infusing dipyridamole. 
When dipyridamole thallium scintigraphy results are considered in conjunction with important clinical parameters (angina, CHF, Q wave on ECG, diabetes mellitus, PVCs), they add significant weight when predicting adverse outcomes for patients with PVD. ${ }^{30}$ Risk stratification may be further enhanced by quantifying the number of areas on thallium imaging that show evidence of redistribution on delayed imaging studies.

A strategy for patients with PVD scheduled for a major vascular procedure may be to conduct a noninvasive stress test, which if negative suggests that surgery can proceed without further testing. ${ }^{31}$ If the imaging study shows evidence of thallium redistribution, the size of the defect should be measured. If in the opinion of the radiologist or cardiologist the defect is small, the risk is low; but if it is moderate to large, the patient may be a candidate for cardiac catheterization and revascularization before having major noncardiac surgery. If the patient is not a candidate for coronary artery revascularization, the previously planned surgery or an alternative surgical procedure should be considered. If the patient is a candidate for coronary vascularization, coronary angiography may be indicated.

It is important to consider the cumulative and relative risks of having these procedures ${ }^{32}$ (Table 10.4). The risk of mortality from coronary angiography is about $1 \%$, and the risk for CABG or PTCA in a patient with PVD is in the range of $6 \%$ to $10 \%$. The risk of delaying noncardiac surgery for testing depends on the reason for the delay and the urgency of the proposed procedure (e.g., a limb that is ischemic or in jeopardy). The risk of mortality for noncardiac surgery after a revascularization procedure is still in the range of $2 \%$ to

\section{Table 10.4. Relative Risk for Further Investigation Versus \\ Proceeding with Noncardiac Surgery in Patients with PVD}

No revascularization prior to noncardiac surgery

Risk of noncardiac procedure

Long-term outcome

\section{Revascularization prior to noncardiac surgery}

Risk of cardiac catherization ( $1 \%$ risk)

Risk of CABG/PTCA (6-10\%)

Risk of delaying surgery

Risk of surgery after revascularization (2-3\%)

Long-term outcome

Perioperative risk without revascularization must be $\geq 15 \%$ for CABG or PTCA to be of benefit.

$\mathrm{PVD}=$ peripheral vascular disease $\mathrm{CABG}=$ coronary artery bypass grafting; PTCA = percutaneous transluminal coronary angioplasty. 
$3 \%$, together with unknown long-term risks of morbidity and mortality. Thus the potential risk of death by further investigating and treating patients with PVD is in the range of about $10 \%$ to $15 \%$. If the risk of the noncardiac procedure without further testing is less, it may be appropriate to proceed with surgery and inform the patient, anesthesiologist, and surgeon of the increased risk. ${ }^{33}$

\section{Medications}

In general, all medications should be continued up to the day of surgery. Monoamine oxidase inhibitors interfere with autonomic function and may cause perioperative hypertension and hypotension. These agents may prolong neuromuscular blockade, inhibit hepatic enzymes, and prolong the action of narcotic drugs. If possible, the medication is discontinued a few weeks before surgery. Beta-blockers and clonidine should be continued until the day of surgery, as there is a possibility of postwithdrawal rebound hypertension. The surgeon should order antibiotic prophylaxis. Most surgeons prefer first-generation cephalosporins (usually cefazolin), starting with the preanesthetic dose and continuing for 24 hours.

Management of anticoagulants may be problematic during the perioperative period. For patients taking Coumadin, the risk of discontinuing anticoagulation must be assessed. For patients with metallic heart valves, Coumadin discontinuation is risky, although continuing Coumadin up to the time of surgery is contraindicated. If it is reasonable to discontinue the Coumadin, it is stopped 3 days before surgery and then reinstituted after surgery. If it is necessary to anticoagulate the patient up to surgery, Coumadin can be stopped 3 days before the operation and the patient treated with intravenous heparin. The heparin infusion is stopped 6 hours before surgery, and Coumadin is reinstituted after surgery. Alternatively, low molecular weight heparin (LMWH) may be used in doses of $\sim 1 \mathrm{mg} / \mathrm{hr}$ every 12 hours adjusted to renal function for 3 days prior to surgery with the last dose administered 12 hours prior to the operation. LMWH or Coumadin may then be reinstituted after surgery. Aspirin, which irreversibly inhibits cyclooxygenase and affects platelet adhesiveness, should be discontinued 7 days before surgery. Other nonsteroidal and nonsalicylate products may be continued up to surgery.

Patients who are at risk for bacterial endocarditis (patients with prosthetic heart valves, previous endocarditis, congenital malformations, hypertrophic cardiomyopathy, and mitral valve prolapse with mitral regurgitation) should receive prophylactic antibiotic coverage (Table 10.5). Bacterial endocarditis prophylaxis is recommended for 


\section{Table 10.5. Bacterial Endocarditis Prophylaxis}

Dental, Oral, Respiratory Tract, or Esophageal Procedures (Followup dose no longer recommended.) Total children's dose should not exceed adult dose.

\section{Standard general prophylaxis for pateints at risk:}

Amoxicillin: Adults, $2.0 \mathrm{~g}$ (children, $50 \mathrm{mg} / \mathrm{kg}$ ) given orally one hour before procedure.

II. Unable to take oral medications:

Ampicillin: Adults, $2.0 \mathrm{~g}$ (children, $50 \mathrm{mg} / \mathrm{kg}$ ) given IM or IV within 30 minutes before procedure.

\section{Amoxicillin/ampicillin/penicillin allergic patients:}

Clindamycin: Adults, $600 \mathrm{mg}$ (children, $20 \mathrm{mg} / \mathrm{kg}$ ) given orally one hour before procedure. -OR-

Cephalexin* or Cefadroxil*: Adults, 2.0 g (children, $50 \mathrm{mg} / \mathrm{kg}$ ) orally one hour before procedure. -OR-

Azithromycin or Clarithromycin: Adults, 500 mg (children 15 $\mathrm{mg} / \mathrm{kg}$ ) orally one hour before procedure.

\section{Amoxicillin/ampicillin/penicillin allergic patients unable to take} oral medications:

Clindamycin : Adults, $600 \mathrm{mg}$ (children, $20 \mathrm{mg} / \mathrm{kg}$ ) IV within 30 minutes before procedure. -OR-

Cefazolin*: Adults, $1.0 \mathrm{~g}$ (children, $25 \mathrm{mg} / \mathrm{kg}$ ) IM or IV within 30 minutes before procedure.

*Cephalosporins should not be used in patients with immediate-type hypersensitivity reaction to penicillins.

\section{Genitourinary/Gastrointestinal Procedures}

\section{High-risk patients:}

Ampicillin plus gentamicin: Amplicillin (adults, 2.0 g; children $50 \mathrm{mg} / \mathrm{kg}$ ) plus gentamicin $1.5 \mathrm{mg} / \mathrm{kg}$ (for both adults and children, not to exceed $120 \mathrm{mg}$ ) IM or IV within 30 minutes before starting procedure; 6 hours later ampicillin (adults, 1.0 g; children, 25 $\mathrm{mg} / \mathrm{kg}$ ) IM or IV, or amoxicillin (adults, $1.0 \mathrm{~g}$; children, $25 \mathrm{mg} / \mathrm{kg}$ ) orally.

\section{High-risk patients allergic to ampicillin/amoxicillin:}

Vancomycin plus gentamicin: Vancomycin (adults, $1.0 \mathrm{~g}$; children, $20 \mathrm{mg} / \mathrm{kg}$ ) IV over $1-2$ hours plus gentamicin $1.5 \mathrm{mg} / \mathrm{kg}$ (for both adults and children, not to exceed $120 \mathrm{mg}$ ) IM or IV. Complete injection/infusion within 30 minutes before starting procedure.

\section{Moderate-risk patients:}

Amoxicillin: Adults, $2.0 \mathrm{~g}$ (children, $50 \mathrm{mg} / \mathrm{kg}$ ) orally one hour before procedure -OR-

Ampicillin: Adults, $2.0 \mathrm{~g}$ (children, $50 \mathrm{mg} / \mathrm{kg}$ ) IM or IV within 30 minutes before starting procedure. 


\section{Table 10.5. (Continued)}

IV. Moderate-risk patients allergic to ampicillin/amoxicillin:

Vancomycin: Adults, $1.0 \mathrm{~g}$ (children $20 \mathrm{mg} / \mathrm{kg}$ ) IV over $1-2$ hours.

Complete infusion within 30 minutes of starting the procedure.

NOTE: For patients already taking an antibiotic, or for other special situations, please refer to the full statement referenced below.

Adapted from Prevention of Bacterial Endocarditis: Recommendations by the American Heart Association by the Committee on Rheumatic Fever, Endocarditis, and Kawasaki Disease. JAMA 1997, 277:17941801, Circulation 1997, 96:358-366, and JADA 1997, 128:1142-1150. Health Care Professionals_-Please refer to these recommendations (endorsed by the American Dental Association and American Society for Gastrointestinal Endoscopy) for more complete information as to which patients and which procedures need prophylaxis.

Source: Reproduced with permission.

http://www.americanheart.org/presenter.jhtml?identifier $=11086$

(c) 2004, American Heart Association.

cardiac conditions that are high (prosthetic valves) or intermediate risk (mitral valve prolapse with regurgitation), for developing endocarditis; and for procedures that may result in bacteremia. Simplified prophylaxis regimens are recommended for dental, gastrointestinal, and genitourinal procedures. There is no need to institute endocarditis prophylaxis for patients with atrial septal defects, post-CABG patients, or patients with pacemakers. ${ }^{34}$

\section{Pulmonary System}

Pulmonary complications are the most common reasons for morbidity among patients undergoing noncardiac surgery (25-50\% of major surgical procedures). Pneumonia, lobar collapse, pneumonitis, atelectasis, and respiratory failure can occur. These complications frequently result in prolonged hospital stays and increased mortality.

A number of reproducible physiologic changes occur with general anesthesia that place patients (especially those with underlying pulmonary disease) at risk of developing a respiratory complication. Predictable changes occur in patterns of ventilation, lung volumes, gas exchange, and pulmonary defense mechanisms. ${ }^{35}$ These changes occur as a result of the procedure itself, the anesthesia, altered sensorium, analgesia, immobility, restrictive bandages, and relative immobility. General anesthesia results in a $20 \%$ decrease in tidal volume, but a compensatory increase in respiratory rate occurs, such that the minute ventilation changes minimally. Lung compliance de- 


\section{Table 10.6. Major Risks for Adverse Pulmonary Outcomes}

Forced expiratory volume in 1 second $\left(\mathrm{FEV}_{1}\right)<1 \mathrm{~L}$

Maximum voluntary ventilation $<50 \%$ of predicted

$\mathrm{PCO}_{2}>90 \mathrm{~mm} \mathrm{Hg}$

$\mathrm{PCO}_{2}<50 \mathrm{~mm} \mathrm{Hg}$

Relative hypoxemia

Upper abdominal and intrathoracic procedures

Prolonged anesthesia ( $>3$ hours)

Cigarette abuse

Obesity ( $>30 \%$ above ideal body weight)

Chronic obstructive pulmonary disease

American Society of Anesthesiologists (ASA) class IV

Age $>70$ years

creases by $33 \%$, and sighing is abolished by the effects of narcotic medications. Total lung capacity and all subdivisions of lung volumes decrease. Vital capacity decreases by half and lasts for 2 weeks after general anesthesia. Most importantly, functional residual capacity (FRC) decreases by up to $40 \%$ after upper abdominal and thoracic procedures. Closing volume $(\mathrm{CV}$, the volume at which airway flow stops during expiration) increases significantly. Under normal circumstances FRC is about $50 \%$ and $\mathrm{CV}$ is about $30 \%$ of total lung capacity, and when these volumes decrease because of general anesthesia the lungs become subject to airway closure, atelactasis, and pneumonia. ${ }^{36}$

General anesthesia results in relative hypoxia because of ventilationperfusion mismatches. Nondependent areas of lung are relatively overventilated and underperfused (dead space), whereas dependent parts of the lung are relatively perfused and underventilated (shunts). Normal pulmonary defense mechanisms (cough and ciliary action) are also impaired during general anesthesia.

Factors that further increase the likelihood of developing pulmonary complications (Table 10.6) include the site of the incision (thoracic and upper abdominal incisions), supine position, prolonged anesthesia ( $>3$ hours), a history of productive cough, cigarette smoking, and fluid overload. Obesity, defined as weighing more than $30 \%$ over ideal body weight, is associated with an increased work of breathing, reduced lung volumes, and hypercapnia; all are associated with an increased risk of complications.

The limits of pulmonary operability have never been clearly defined. There are no studies that demonstrate the level of forced expiratory volume in 1 second $\left(\mathrm{FEV}_{1}\right)$ below which a person is at increased risk, nor is there consensus about indications for preoperative 
pulmonary function testing. ${ }^{37}$ An $\mathrm{FEV}_{1}$ of less than $2 \mathrm{~L}$ is associated with an increased risk of pulmonary complications, and patients with an $\mathrm{FEV}_{1}$ value as low as $1 \mathrm{~L}$ may be more likely to require ventilator support and develop pulmonary complications.

There are no clear guidelines for estimating potential risk of developing pulmonary complications prior to surgery. Chest radiographs may be useful in high-risk patients. There are no clear indications for ordering pulmonary function tests. These tests may help in defining the severity of underlying pulmonary disease, and thus may help estimate the potential risk for complications. For patients who are to have cardiac surgery, pneumonectomy, or esophagectomy, more sophisticated testing such as quantitative ventilation-perfusion scanning, diffusing capacity studies, and estimation of maximum oxygen consumption during exercise may be indicated.

For patients with pulmonary disease, it may be possible to reduce complications by instructing them to stop cigarette smoking at least 8 weeks before surgery, ${ }^{38}$ which results in enhanced mucociliary transport mechanisms, reduced secretions, less bronchospasm, and reduced levels of circulating carboxyl hemoglobin. Optimal bronchodilation prior to surgery may require home or inpatient nebulizer treatments and use of steroids for patients with asthma. Incentive spirometry has been shown to reduce the incidence of pulmonary complications and the length of hospital stay. Emphasis is placed on general conditioning, nutrition, and psychological preparation if a person is likely to spend some time in the intensive care unit after surgery. Anesthesiologists are aware of the need to minimize anesthesia time, use intermittent hyperinflation, and control secretions. Postoperatively, patients should be encouraged to get out of bed early, take deep breaths, use the incentive spirometer, and cough. Narcotic and analgesic medications should be administered in doses titrated to obtain analgesic effects without clouding the sensorium. Because cardiac and pulmonary complications occur for up to 7 days after surgery, medical consultants should closely monitor patients during this stressful time.

\section{Hematologic System}

\section{Hemoglobin and Hematocrit}

The optimal levels of hemoglobin and hematocrit for surgery traditionally have been $\geq 10 \mathrm{~g} / \mathrm{dL}$ and $30 \%$, respectively. Consensus conference reports have now recognized the increasing evidence of the safety of transfused blood products and the excellent outcomes of surgery on anemic patients; it is therefore concluded that no single 
criterion can be used to support preoperative blood transfusion. ${ }^{39}$ There is good evidence that surgical procedures can be done in patients with hemoglobin levels above $8 \mathrm{~g} / \mathrm{dL}$ and in patients with hemoglobin levels as low as $6 \mathrm{~g} / \mathrm{dL}$ if blood loss is less than $500 \mathrm{~mL}$. The risks associated with blood transfusion are low; there is a less than $1 \%$ risk of transfusion mortality associated with the human immunodeficiency virus (HIV) and a less than $0.3 \%$ risk of acquiring hepatitis C (since testing became available during the 1990s); the risk of hemolytic reactions or congestive cardiac failure is also low.

Higher hemoglobin levels are advisable for older patients, those likely to experience significant blood loss, and patients with coronary artery or cerebrovascular disease. Lower levels of hemoglobin are acceptable for patients with chronic anemia with compensated intravascular volumes. When possible, transfusion is done preoperatively, as transfusion reaction signs may be obliterated under general anesthesia. The more widespread use of autologous blood, cell savers, and erythropoietin has greatly reduced the need for transfusion with banked blood. ${ }^{40}$ The hematocrit level for optimal oxygen delivery to tissues and rheology is around $45 \%$. Patients with polycythemia and erythrocytosis are more likely to bleed or have blood clots and should be phlebotomized to hematocrits of around $45 \%$ to $50 \%$ before elective surgery.

\section{Platelets}

Patients with thrombocytopenia rarely bleed until platelet counts are below 100,000 cells $/ \mu \mathrm{L}$, and the risk of bleeding is proceduredependent for patients with platelet counts between 50,000 and $100,000 / \mu \mathrm{L} .{ }^{41}$ Platelet counts below $50,000 / \mu \mathrm{L}$ are associated with an increased risk of bleeding. Qualitative platelet functioning can be impaired even when platelet counts are normal. Medications including nonsteroidal antiinflammatory drugs (NSAIDs), aspirin, tricyclic antidepressants, alcohol, and even beta-blockers can impair platelet adhesion. Patients with uremia, liver disease, alcoholism, and leukemia may also develop qualitative platelet disorders. For these patients it may be useful to determine the bleeding time prior to surgery.

\section{Coagulation Disorders}

Coagulation disorders are rare, as a loss of $80 \%$ of clotting factor levels is required to prolong the prothrombin time (PT) or partial thromboplastin time (PTT). The most common disorder affecting clotting factor production is severe liver disease. Patients with prolonged PTs and PTTs can be managed intraoperatively with transfusions of fresh frozen plasma and whole blood. For patients with 
known or suspected acquired factor deficiency, a hematologist should be consulted for further investigation, recommendations, and treatment perioperatively.

\section{Deep Venous Thrombosis}

All surgical patients are at risk for developing deep venous thrombosis (DVT) (see Chapter 7). This risk is increased for elderly patients, the obese, cigarette smokers, cancer patients, patients who are having long procedures, those with previous venous disease, and those with a history of CHF. The risk of developing a postoperative DVT depends on the type of surgical procedure and the presence of risk factors. The optimal modality for DVT prophylaxis is controversial. LMWH (40 mg/day or $30 \mathrm{mg}$ q12h) significantly reduces the incidence of DVT and pulmonary embolism in patients undergoing general surgical procedures, such as urologic or gynecologic surgery, surgery for elective hip or knee replacement or hip fracture, and other orthopedic procedures including trauma surgery. LMWH in doses of $40 \mathrm{mg}$ per day or $30 \mathrm{mg}$ every 12 hours significantly reduces the incidence of postoperative DVT. ${ }^{42}$ It is unclear if LMWH is superior to warfarin (Coumadin) for prophylaxis of DVT for major surgical procedures, including orthopedic surgery. LMWH is expensive and not without side effects (thrombocytopenia, bleeding). Dosing of LMWH must be adjusted according to creatinine clearance. Elderly patients should be carefully monitored for bleeding complications. LMWH prophylaxis is not recommended for patients who are to have neurosurgical procedures or spinal anesthesia, because of an increased risk of bleeding that may compromise neurologic functioning. ${ }^{43}$ Neurosurgical patients or patients undergoing spinal cord surgical procedures should have prophylaxis with intermittent compression or elastic stockings, as there is no risk for hemorrhagic complications. A decision to institute pharmacologic prophylaxis should be made by the neurosurgeon.

Multicenter trials comparing warfarin and LMWH are currently under way. Consensus conference opinion supports the use of LMWH (30 mg enoxaparin SC q12h), heparin (5000 IU SC q12h), or warfarin $(10 \mathrm{mg}$ po on the night of surgery followed by $5 \mathrm{mg}$ po qhs, titrating the dose to achieve an international normalized ratio of 2-3) for prophylaxis of DVT for general surgery and orthopedic surgical patients. The optimal duration of DVT prophylaxis is controversial. Some would recommend that LMWH or warfarin be continued for 6 weeks to 2 months after surgery. At a minimum DVT prophylaxis should be continued until the patient is ambulatory for functional distances. 


\section{Liver Disease}

For patients with liver disease, the likelihood of developing complications is related to the degree of hepatic compromise. ${ }^{44}$ Patients with acute viral hepatitis and elevated liver enzyme levels, determined by liver function tests (LFTs), should not have surgery until about 4 weeks after the LFTs are normalized. Most surgical procedures cause elevated LFTs because of hypoxia, hypercarbia, traction, reduced blood flow, and portal hypotension induced by anesthetic agents. The major perioperative complications in patients with liver disease are bleeding, infection, and renal insufficiency (especially in patients with obstructive jaundice secondary to malignant disease). Patients with liver disease are likely to have a poor prognosis if they have hypoalbuminemia, a prolonged prothrombin time, ascites, esophageal varices, or encephalopathy. These patients should be managed aggressively with the help of a gastroenterologist using mannitol, lactulose, hyperalimentation, fresh frozen plasma, vitamin $\mathrm{K}$, spironolactone, and other diuretics.

\section{Endocrine Disorders}

The most common endocrine disorder encountered during the perioperative period is diabetes mellitus. Surgical mortality for patients with diabetes is greater among those who have concomitant cardiac disease, those who are having peripheral vascular surgery, and those with end-organ damage (autonomic neuropathy or nephropathy) as a result of their diabetes. Autonomic cardiac neuropathy may result in reduced ability to perceive postoperative chest pain, and these patients may be unable to mount an adequate cardiac response to the myocardial suppression due to anesthesia. Patients with diabetes are more likely to develop urinary retention, gastric retention, and pulmonary aspiration. Surgery places an additional strain on the diabetic patient, resulting in wide swings in blood glucose levels, volume shifts, electrolyte abnormalities (most frequently hypo- and hyperkalemia), and changes in acid-base status. ${ }^{45}$

The goal of management is to control blood glucose levels throughout the period of surgical stress. A number of opposing factors are involved. Usually patients are not eating well, which reduces the requirement for insulin, and they are inactive, increasing insulin requirements. During surgery there is an outpouring of catecholamines, glucagon, and cortisol, which dramatically increase insulin requirements. The net effect is the frequently encountered hyperglycemia during surgery. In general, the goal is to keep the blood glucose level below $250 \mathrm{mg} / \mathrm{dL}$. For patients who are insulin-dependent, one half 
to two thirds of their intermediate-acting insulin should be administered on the morning of surgery and fingerstick glucose levels monitored every 2 hours during surgery, with sliding-scale regular insulin coverage. For patients with "brittle" diabetes, better control may be achieved by starting an insulin infusion a few hours before surgery. The usual rate of infusion is 2 units of insulin per hour, but this dosage can be adjusted depending on the serum glucose levels. To avoid hypoglycemia, a dextrose infusion is administered simultaneously in the other arm. The infusion rates are adjusted based on fingerstick glucose levels. For patients with diabetes taking oral hypoglycemic agents, the oral agent is stopped on the day before surgery and the patient managed with sliding-scale insulin similarly to the patient with insulin-dependent diabetes.

\section{Steroid Use}

Patients who are currently taking corticosteroids may be at risk of hypothalamic-pituitary-adrenal axis insufficiency when faced with the stress of surgery. Published case reports describe cardiovascular collapse among patients with steroid-related adrenal insufficiency. Patients who are currently taking steroids, who have used longacting steroids, or who have taken steroids for more than a week during the previous year may be at increased risk of iatrogenic axis suppression. Consideration should be given to treating these patients with "stress-dose" intravenous steroids during surgery. One critical review of the literature on stress-dose steroids reported that few studies fulfilled the diagnostic criteria for iatrogenic adrenal insufficiency. ${ }^{46}$ Furthermore, studies of renal allograft patients who had adrenal functioning testing reported that patients who had been taking prednisone for long periods had normal adrenal responses to adrenocorticotropic hormone (ACTH) stimulation, suggesting that adrenal function was preserved. For patients who are at risk for adrenal axis suppression, it may be useful to assess adrenal gland function. If the patient's cortisol level doubles over a fasting level 1 hour after administering 250 $\mu \mathrm{g}$ of ACTH intravenously or intramuscularly, or if any cortisol level is in the range of 17 to $20 \mu \mathrm{g} / \mathrm{dL}$, adrenal function is likely to be intact. If it is decided to treat the patient empirically with stress-dose steroids, hydrocortisone sodium succinate (Solu-Cortef) or equivalent $100 \mathrm{mg}$ IV q6h should be given for 24 hours, tapering the dose by $50 \%$ until the patient can take the usual dose of steroids.

\section{Renal System}

New-onset renal insufficiency during the perioperative period is associated with significant morbidity and mortality. A meta-analysis 
study reported that the only consistent predictors of postoperative acute tubular necrosis were preoperative elevations of creatinine and blood urea nitrogen (BUN) and patients having a major vascular procedure. ${ }^{47}$ Patients who are volume-depleted, elderly, septic, or having a major procedure are at increased risk for developing perioperative acute tubular necrosis. Morbidity and mortality result from sepsis, coagulopathy, and volume and electrolyte disturbances. Anesthesiologists have a heightened level of concern about avoiding this complication, especially in patients with a suspected creatinine clearance of less than 50\%. Patients on chronic hemodialysis or peritoneal dialysis can be operated on safely, but dialysis should be completed 6 hours or more before surgery because of the anticoagulant effects of heparin added to the dialysate.

\section{Nutrition}

Protein-calorie undernutrition results in increased surgical morbidity and mortality. Surgery may transform mild undernutrition into severe malnutrition, which if it occurs is associated with impaired wound healing, immunodeficiencies, and a reduced ability to resist infection. Older patients are especially at risk for undernutrition after surgery because of coexistent chronic medical illnesses (e.g., congestive heart failure, chronic lung disease), functional impairments, coexistent dementia/postoperative delirium, and surgery-related factors (e.g., nil per mouth, liquid diet).

Patients who have multiple trauma or pancreatitis or who are unable to eat for 7 to 10 days are most likely to develop perioperative malnutrition. The Veterans Administration Parenteral Nutrition Cooperative Study found that mortality and complication rates among patients who received total parenteral nutrition (TPN) were similar to those who did not. ${ }^{48}$ The authors concluded that TPN should be considered for severely malnourished patients or those whose gastrointestinal tract was to be rested for 7 to 10 days if previously normally nourished or for 5 days in previously malnourished patients. The exact optimal duration of nutritional support is not well established; however, low-risk nutritional supplementation (oral supplements) make intuitive sense for all perioperative patients until adequate nutritional intake is established.

\section{Conclusion}

Family physicians are increasingly being requested to participate in the care of patients during the perioperative period. In general, sur- 
gical and anesthesia outcomes have greatly improved, even among elderly patients in good medical condition. There remain, however, categories of patients who are at high risk for adverse outcomes, especially those with comorbid cardiovascular disease, those having peripheral vascular surgery, and those at risk for developing pulmonary complications, sepsis, or renal failure. The medical care of the surgical patient is highly individualized, with a goal of bringing the person to the operating room in the best condition possible and then not abandoning the patient after surgery.

\section{References}

1. Milamed DR, Hedley-Whyte J. Contributions of the surgical sciences to a reduction of the mortality rate in the United States for the period 1968 to 1988. Ann Surg 1994;219:94-102.

2. Khuri SF, Daley J, Henderson W, et al. The National Veterans Administration Surgical Risk Study: risk adjustment for the comparative assessment of the quality of surgical care. J Am Coll Surg 1995;180:519-31.

3. King MS. Preoperative evaluation. Am Fam Physician 2000;62:387-93.

4. Adrenal dysfunction and steroid use. In: Adler AG, Merli GJ, McElwain GE, Martin JH, eds. Medical evaluation of the surgical patient. Philadelphia: WB Saunders, 1985;101-17.

5. MacPherson, DS. Preoperative laboratory testing: should any tests be "routine" before surgery? Med Clin North Am 1993;77:289-306.

6. More preoperative assessment by physicians and less by laboratory tests [editorial]. N Engl J Med 2000;342:204-5.

7. Fischer S. Cost-effective preoperative evaluation and testing. Chest 1999;115(suppl 5):96S-100S.

8. MacPherson DS, Snow R, Lofgren RP. Preoperative screening: value of previous tests. Ann Intern Med 1990;113:969-73.

9. Farrow SC, Fowkes FGR, Lunn JN, et al. Epidemiology in anesthesia. II. Factors affecting mortality in hospital. Br J Anaesth 1982;54:811-7.

10. Dripps RD. A new classification of physical status. Anesthesiology 1963;24:111.

11. Goldman L. Cardiac risks and complications of non-cardiac surgery. Ann Intern Med 1983;98:504-13.

12. Belzberg H, Rivkind AI. Preoperative cardiac preparation. Chest 1999; 115(suppl 5):82S-95S.

13. Detsky AS, Abrams HB, McLaughlin JR. Predicting cardiac complications in patients undergoing noncardiac surgery. J Gen Intern Med 1986;1:211-9.

14. Jeffrey CC, Kunsman J, Cullen DJ, Brewster DC. A prospective evaluation of cardiac risk index. Anesthesiology 1983;58:462-4.

15. Hollenberg SM. Preoperative cardiac risk assessment. Chest 1999; 115(Suppl 5):51S-7S.

16. Eagle KA, Brundage BH, Chaitman BR, et al. Guidelines for perioperative cardiovascular evaluation for noncardiac surgery; report of the 
American College of Cardiology/American Heart Association Task Force on Practice Guidelines (Committee on Perioperative Cardiovascular Evaluation for Noncardiac Surgery). J Am Coll Cardiol 1996;27: 910-48.

17. American College of Physicians. Clinical guideline, Part I. Guidelines for assessing and managing the perioperative risk from coronary artery disease associated with major noncardiac surgery. Ann Intern Med 1997;127:309-12.

18. Crawford ES, Morris GC, Howell JF, Flynn WF, Moorhead DT. Operative risk in patients with previous coronary artery bypass. Ann Thorac Surg 1978;26:215-21.

19. Mangano DT, Layug EL, Wallace A, Tateo I. Effect of atenolol on mortality and cardiovascular morbidity after noncardiac surgery. N Engl J Med 1996;335:1713-20.

20. Higham H, Handa A, Hands LJ, et al. Slowing the heart saves lives: advantage of perioperative beta-blockade. Br J Surg 2000;87(12):1736-7.

21. Weitz HH. Non-cardiac surgery in the elderly patient with cardiovascular disease. Clin Geriatr Med 1990;6:511-29.

22. Wolfsthal SD. Is blood pressure control necessary before surgery? Med Clin North Am 1993;77:349-63.

23. O’Kelly B, Browner WS, Massie B, Tubau J, Ngo L, Mangano DT. Ventricular arrhythmias in patients undergoing noncardiac surgery. JAMA 1992;268:217-21.

24. Weitz HH, Goldman L. Non-cardiac surgery in the patient with heart disease. Med Clin North Am 1987;71:413-32.

25. O'Keefe JH, Shub C, Rettke SR. Risk of non-cardiac surgical procedures in patients with aortic stenosis. Mayo Clin Proc 1989;64:400-5.

26. Wong T, Detsky AS. Having peripheral vascular surgery. Ann Intern Med 1992;116:743-53.

27. Fleisher LA, Barash PG. Preoperative evaluation of the cardiac patient for noncardiac surgery. Yale J Biol Med 1993;66:385-95.

28. Mangano DT, Hollenberg M, Fegert G, et al. Perioperative myocardial ischemia in patients undergoing noncardiac surgery. I. Incidence and severity during the 4 day perioperative period. J Am Coll Cardiol 1991;17:843-50.

29. Botvinick EH, Dae MW. Dipyridamole perfusion scintigraphy. Semin Nucl Med 1991;21:242-65.

30. Lette J, Waters D, Cerino M, Picard M, Champagne P, Lapointe J. Preoperative coronary artery disease risk stratification based on dipyridamole imaging and a simple three-step, three-segment model for patients undergoing noncardiac vascular surgery or major general surgery. Am J Cardiol 1992;69:1553-8.

31. Fleisher LA, Barash PG. Preoperative cardiac evaluation for noncardiac surgery: a functional approach. Anesth Analg 1992;74:586-98.

32. Potyk DK. Cardiac evaluation and risk reduction in patients undergoing major vascular operations. West J Med 1994;161:50-6.

33. Goldman L. Cardiac risk for vascular surgery (editorial comment). J Am Coll Cardiol 1996;27(4):799-802. 
34. Dajani AS, Taubert KA, Wilson W, et al. Prevention of bacterial endocarditis. Recommendations of the American Heart Association. JAMA 1997;277:1794-801.

35. Tisi GM. Preoperative identification and evaluation of a patient with lung disease. Med Clin North Am 1987;71:399-412.

36. Ferguson MK. Preoperative assessment of pulmonary risk. Chest 1999;115(suppl 5):58S-63S.

37. Zibrak JD, O'Donnell CR. Indications for preoperative pulmonary function testing. Clin Chest Med 1993;14:227-36.

38. Warner MA, Offord KP, Warner ME, Lennon RI, Conover MA, Jansson-Schumacher U. Role of preoperative cessation of smoking and other factors in postoperative pulmonary complications: a blinded prospective study of coronary artery bypass patients. Mayo Clin Proc 1989;64:609-16.

39. Carson JL, Willett LR. Is a hemoglobin of $10 \mathrm{~g} / \mathrm{dl}$ required for surgery? Med Clin North Am 1993;77:335-47.

40. Konishi T, Ohbayashi T, Kaneko T, Ohki T, Saitou Y, Yamato Y. Preoperative use of erythropoietin for cardiovascular operations in anemia. Ann Thorac Surg 1993;56:101-3.

41. Hematology. In: Adler AA, Merli GJ, McElwain GE, Martin JH, eds. Medical evaluation of the surgical patient. Philadelphia: WB Saunders, $1985 ; 44-50$.

42. Ament PW, Bertolino JG. Enoxaparin in the prevention of deep venous thrombosis. Am Fam Physician 1994;50:1763-8.

43. Clagett GP, Anderson FA, Geerts W, et al. Prevention of venous thromboembolism. Chest 1998;114(suppl 5):531S-60S.

44. Brown FH, Shiau YF, Richter GC. Anesthesia and surgery in the patient with liver disease. In: Goldmann DR, Brown FH, Levy WK, et al, eds. Medical care of the surgical patient: a problem-oriented approach to management. Philadelphia: Lippincott, 1982;326-42.

45. Ockert DBM, Hugo JM. Diabetic complications with special anaesthetic risk. S Afr J Surg 1992;30:90-4.

46. Salem M, Tainsh RE, Bromberg J, Loriaux DL, Chernow B. Perioperative glucocorticoid coverage: a reassessment 42 years after emergence of a problem. Ann Surg 1994;219:416-25.

47. Novis BK, Roizen MF, Aronson S, Thisted RA. Association of preoperative risk factors with postoperative acute renal failure. Anesth Analg 1994;78:143-9.

48. Buzby GP. Overview of randomized clinical trials of total parenteral nutrition for malnourished surgical patients. World J Surg 1993;17:173-7. 